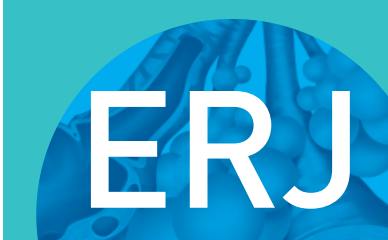

open research

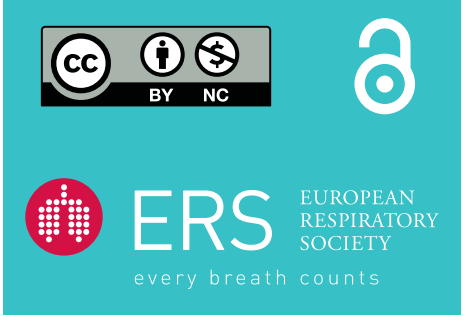

\section{Steroid-sparing effects of benralizumab in patients with eosinophilic granulomatosis with polyangiitis}

\author{
To the Editor:
}

Eosinophilic granulomatosis with polyangitis (EGPA) is a rare, anti-neutrophil cytoplasmic antibody (ANCA)-associated vasculitis characterised by asthma, chronic rhinosinusitis and blood eosinophilia, which may be accompanied by neurological, cardiac, cutaneous and renal involvement [1]. Oral corticosteroids (OCS) are the most frequently used drugs to control eosinophilic inflammation and symptoms. Persistent symptoms or relapses are common, however, and many patients are at risk of developing long-term complications from systemic steroid therapy [2]. The ability of other immunosuppressant agents to achieve consistent disease control or to reduce maintenance OCS (mOCS) requirements appears to be limited [3].

Interleukin (IL)-5 is a key cytokine implicated in the proliferation, maturation and differentiation of eosinophils, and clinical trials have reported a beneficial and steroid-sparing effect of the subcutaneous anti-IL-5 monoclonal antibody (mAb) mepolizumab in patients with EGPA [4]. We have previously reported on the effect of the intravenous anti-IL-5 mAb reslizumab in EGPA [5]. Here, we present our experience with benralizumab, which mediates eosinophil apoptosis via binding to IL- 5 receptor- $\alpha$, and its effect on patient-reported outcomes and OCS requirements in patients with EGPA.

Patients with established EGPA based on the American College of Rheumatology 1990 criteria [6] and on mOCS therapy were commenced on benralizumab $30 \mathrm{mg}$ administered by subcutaneous injections every 8 weeks, with the first three doses given every 4 weeks. All patients attended a dedicated EGPA clinic run jointly between respiratory and rheumatology physicians, and all patients gave written informed consent. Ethical approval was gained from the London Bloomsbury Research Ethics Committee (915/LO/0886). Patient-reported outcomes were assessed by the Birmingham Vasculitis Activity Score (BVAS), the Asthma Control Questionnaire (ACQ), the Asthma Quality of Life Questionnaire (AQLQ) and the Sino-Nasal Outcome Test (SNOT)-22. Lung function, blood eosinophils, white blood cell count, C-reactive protein $(\mathrm{CRP})$, troponin $\mathrm{T}$, renal function, fractional exhaled nitric oxide $\left(F_{\mathrm{ENO}}\right)$, mOCS dose and exacerbation rate were recorded at baseline, 24 weeks and 48 weeks of treatment. All patients had confirmed adherence to prednisolone via paired blood prednisolone/cortisol levels.

11 patients (six female) with a mean \pm SD age of $50 \pm 14$ years had completed 24 weeks of treatment by the time of this analysis; nine had completed 48 weeks of treatment. All 11 patients had severe eosinophilic asthma and paranasal sinus involvement. Two (18\%) had cardiac, one (9\%) dermatological and one (9\%) neurological involvement. ANCA were positive in four (36\%); three (27\%) had histopathological evidence of eosinophilic vasculitis. Five (46\%) patients had received treatment with other immunosuppressive drugs (mycophenolate mofetil, methotrexate and azathioprine); three (36\%) had previously failed treatment with mepolizumab, whilst one patient had failed both mepolizumab and reslizumab, based on ongoing OCS requirements and high symptom score, and assessed by a multidisciplinary team review.

@ERSpublications

Benralizumab reduces oral corticosteroid requirements in patients with EGPA and leads to improved patient-reported outcome measures https://bit.ly/2GI0vhf

Cite this article as: Nanzer AM, Dhariwal J, Kavanagh J, et al. Steroid-sparing effects of benralizumab in patients with eosinophilic granulomatosis with polyangiitis. ERJ Open Res 2020; 6: 00451-2020 [https://doi.org/10.1183/23120541.00451-2020].

This article has been revised according to the erratum published in ERJ Open Res 2021; 7: 50451-2020 [https:// doi.org/10.1183/23120541.50451-2020].

Copyright OERS 2020. This article is open access and distributed under the terms of the Creative Commons Attribution Non-Commercial Licence 4.0. 
At the start of benralizumab therapy, patients were taking a median (interquartile range) dose of 15 (10-20) mg mOCS daily, and had evidence of poor symptom control (ACQ 2.13 \pm 0.98 , AQLQ 4.15 \pm 1.41 and BVAS 7.91 \pm 3.27$)$ and persistent airway inflammation, with a $F_{\mathrm{ENO}}$ of $38(25-77) \mathrm{ppb}$ and mean blood eosinophil count of $0.2(0.1-0.7) \times 10^{9}$ cells per L. Following 24 weeks of treatment, there was a median reduction in mOCS of $50 \%$ and eight $(73 \%)$ out of 11 patients were able to reduce their dose by $\geqslant 50 \%$. Amongst the nine patients who had completed 48 weeks of treatment, the median reduction in mOCS was $65 \%$ and eight $(89 \%)$ were able to reduce their dose by $\geqslant 50 \%$. The median prednisolone dose was reduced from $15(10-20)$ to $5(5-10) \mathrm{mg} \mathrm{mg}$ at 24 weeks and $5(1-6.5) \mathrm{mg}$ at 48 weeks $(\mathrm{p}=0.0018)$ (figure 1a). Blood eosinophil counts were totally depleted after 24 and 48 weeks of treatment. There was no significant difference and no change from baseline to 24 and 48 weeks in white blood count $(9.75 \pm 2.39,7.71 \pm 2.94$ and $7.16 \pm 2.34 \times 10^{9}$ cells per L), CRP (5 (1-7), 7.71 \pm 2.94 and $7.16 \pm 2.34 \mathrm{mg} \cdot \mathrm{L}^{-1}$ ), troponin T (8 (5-11), 7 (5-10) and $\left.6(5-7) \mathrm{ng} \cdot \mathrm{L}^{-1}\right)$ or creatinine $\left(78.8 \pm 16,77.9 \pm 18.8\right.$ and $76.6 \pm 19.4 \mu \mathrm{mol} \cdot \mathrm{L}^{-1}$, respectively).

A significant improvement in BVAS from $7.91 \pm 3.27$ at baseline to $3.45 \pm 2.52$ at 24 weeks $(\mathrm{p}=0.0001)$ and $3.44 \pm 2.88$ at 48 weeks $(\mathrm{p}=0.0007)$ was recorded. The ACQ changed from $2.13 \pm 0.98$ at baseline to $1.73 \pm 1.57$ at 24 weeks $(\mathrm{p}=0.47)$ and $1.03 \pm 0.71$ at 48 weeks $(\mathrm{p}=0.012)$ (figure $1 \mathrm{~b}$ and $\mathrm{c})$. AQLQ scores changed from $4.15 \pm 1.41$ at baseline to $4.96 \pm 1.63$ at 24 weeks $(\mathrm{p}=0.13)$ and $5.5 \pm 1.27$ at 48 weeks $(\mathrm{p}=0.013)$. We recorded an improvement in the SNOT-22 questionnaire from $5.7 \pm 20.8$ at baseline to $35 \pm 20.7$ at 24 weeks $(\mathrm{p}=0.005)$ and $20.9 \pm 12.5$ at 48 weeks $(\mathrm{p}=0.004)$.

No significant changes were observed in absolute forced expiratory volume in $1 \mathrm{~s}\left(\Delta 0.2 \pm 0.3 \mathrm{~L} \cdot \mathrm{s}^{-1}\right.$ at 24 weeks; $\Delta 0.3 \pm 0.4 \mathrm{~L} \cdot \mathrm{s}^{-1}$ at 48 weeks). $F_{\mathrm{ENO}}$ did not significantly change from baseline at 24 weeks $(\Delta$ $6.5 \pm 48 \mathrm{ppb})$ or at 48 weeks $(\Delta-22 \pm 40 \mathrm{ppb})$. No increase in frequency of exacerbations was seen. Benralizumab was well tolerated and no treatment-limiting adverse effects were recorded.

EGPA is a rare but severe systemic illness for which few therapeutic options exist. Hitherto, patients have been treated with high-dose glucocorticosteroids and other immunosuppressants, drugs with considerable side-effect profiles [7]. Inhibiting eosinophilic inflammation in a more targeted manner via blockade of the IL-5 pathway has been a focus of novel therapeutic options in both severe asthma and EGPA. To date, mepolizumab remains the only Food and Drug Administration-approved therapy for EGPA after it was found to be safe and effective in reducing corticosteroid dose and disease relapse [4]. However, persistent tissue eosinophilia despite mepolizumab has been reported and $47 \%$ of subjects in the mepolizumab group of the phase 3 trial did not achieve remission [8]. There is sound rationale that benralizumab, with its unique eosinophil-depleting properties, may offer improved outcomes in EGPA in which multiorgan eosinophilic infiltration is believed to drive morbidity and mortality. Our data demonstrate that EGPA patients treated with benralizumab have substantially reduced mOCS requirements, and significant improvements in patient related outcome measures as early as 24 weeks and maintained up to 48 weeks of treatment. It is noteworthy that our EGPA patient cohort is of the asthma phenotype with foremost airway and dominant sinonasal disease, and a lower prevalence of ANCA positivity and vasculitic features.

Our results are in line with other recently published findings [9] as well as our earlier experience with reslizumab [5]. Important limitations of our data include the relatively small cohort described and the absence of a control arm inherent in any open-label observational report.

In summary, we report significant reductions in mOCS requirements and improved measures of disease control following benralizumab therapy in patients with EGPA. Further research exploring the mechanism(s)
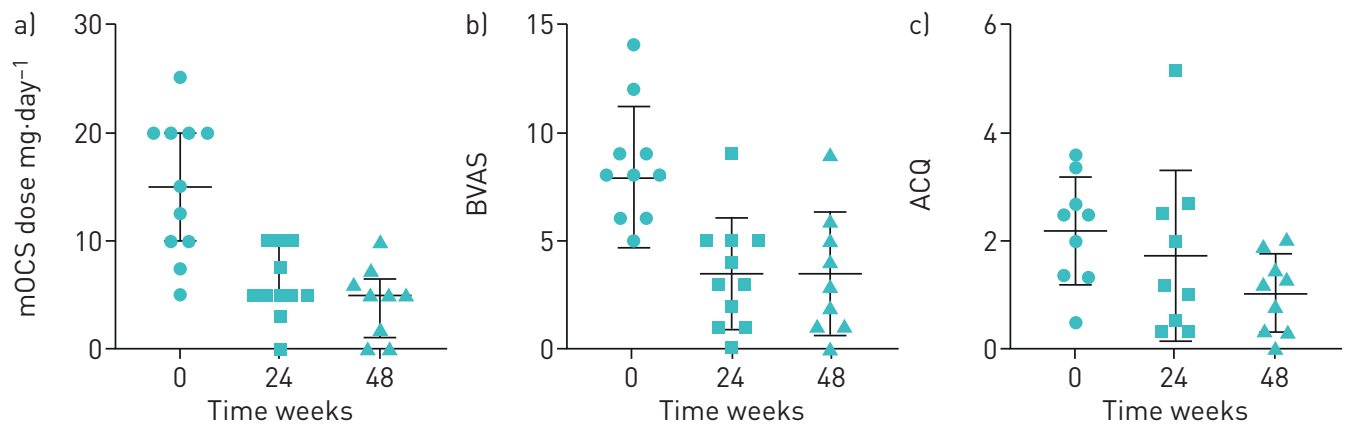

FIGURE 1 Reduction from baseline to 48 weeks in a) maintenance oral corticosteroid (mOCS) dose ( $p=0.0018$ ), b) Birmingham Vasculitis Activity Score (BVAS) ( $p=0.0007$ ) and c) Asthma Control Questionnaire score (ACQ) $(p=0.012$ ). Data are presented as a) median (interquartile range) and $b$ and $c$ ) mean \pm SD. 
of residual disease in eosinopenic patients treated with benralizumab is needed and must compliment upcoming prospective controlled trials of this therapy in EGPA.

Alexandra M. Nanzer $\oplus^{1,2}$, Jaideep Dhariwal $\oplus^{1}$, Joanne Kavanagh ${ }^{1,2}$, Andrew Hearn ${ }^{1,2}$, Mariana Fernandes ${ }^{1}$, Louise Thomson ${ }^{1}$, Cris Roxas ${ }^{1}$, Linda Green ${ }^{1}$, Grainne D'Ancona ${ }^{1}$, Sangita Agarwal $^{3}$, Brian D. Kent ${ }^{1}$ and David J. Jackson $\circledast^{1,2}$

${ }^{1}$ Guy's and St Thomas' NHS Foundation Trust, Thoracic Medicine, Guy's Severe Asthma Service, London, UK. ${ }^{2}$ School of Immunology \& Microbial Sciences, King's College London, London, UK. ${ }^{3}$ Dept of Rheumatology, Guy's and St Thomas' NHS Foundation Trust, London, UK.

Correspondence: Alexandra M. Nanzer, Guy's and St Thomas' NHS Foundation Trust, Thoracic Medicine, Guy’s Severe Asthma Service, Guy’s Hospital, Great Maze Pond, London, SE1 9RT, UK. E-mail: alexandra.nanzerkelly@gstt.nhs.uk

Received: 30 June 2020 | Accepted after revision: 17 Aug 2020 | First published: 10 Nov 2020

Conflict of interest: A.M. Nanzer reports personal fees from AstraZeneca and Napp outside the submitted work. J. Dhariwal has nothing to disclose. J. Kavanagh has nothing to disclose. A. Hearn has nothing to disclose. M. Fernandes has nothing to disclose. L. Thomson has nothing to disclose. C. Roxas has nothing to disclose. L. Green has nothing to disclose. G. D'Ancona reports personal fees from Chiesi, AstraZeneca, GSK and Napp outside the submitted work. S Agarwal has nothing to disclose. B.D. Kent reports personal fees from AstraZeneca and GSK outside the submitted work. D.J. Jackson reports personal fees from AstraZeneca, GSK, Teva, Sanofi and Novartis outside the submitted work.

\section{References}

1 Comarmond C, Pagnoux C, Khellaf M, et al. Eosinophilic granulomatosis with polyangiitis (Churg-Strauss): clinical characteristics and long-term followup of the 383 patients enrolled in the French Vasculitis Study Group cohort. Arthritis Rheum 2013; 65: 270-281.

2 Lefebvre P, Robitaille MN, Albers F, et al. Burden of systemic glucocorticoid-related complications in severe asthma. Curr Med Res Opin 2017; 33: 57-65.

3 Puechal X, Pagnoux C, Baron G, et al. Non-severe eosinophilic granulomatosis with polyangiitis: long-term outcomes after remission-induction trial. Rheumatology (Oxford) 2019; 58: 2107-2116.

4 Wechsler ME, Akuthota P, Jayne D, et al. Mepolizumab or placebo for eosinophilic granulomatosis with polyangiitis. N Engl J Med 2017; 376: 1921-1932.

5 Kent BD, d'Ancona G, Fernandes M, et al. Oral corticosteroid-sparing effects of reslizumab in the treatment of eosinophilic granulomatosis with polyangiitis. ERJ Open Res 2020; 6: 00311-2019.

6 Masi AT, Hunder GG, Lie JT, et al. The American College of Rheumatology 1990 criteria for the classification of Churg-Strauss syndrome (allergic granulomatosis and angiitis). Arthritis Rheum 1990; 33: 1094-1100.

7 Groh M, Pagnoux C, Baldini C, et al. Eosinophilic granulomatosis with polyangiitis (Churg-Strauss) (EGPA) Consensus Task Force recommendations for evaluation and management. Eur J Intern Med 2015; 26: 545-553.

8 Ortega HG, Liu MC, Pavord ID, et al. Mepolizumab treatment in patients with severe eosinophilic asthma. $N$ Engl J Med 2014; 371: 1198-1207.

9 Padoan R, Chieco Bianchi F, Marchi MR, et al. Benralizumab as a glucocorticoid-sparing treatment option for severe asthma in eosinophilic granulomatosis with polyangiitis. J Allergy Clin Immunol Pract 2020; 8: 3225-3227. 